\title{
Mechanical thrombectomy for intra-procedural ischemic stroke during transcatheter aortic valve implantation
}

\author{
Krzysztof Pyra ${ }^{1}$, Maciej Szmygin ${ }^{1}$, Krzysztof Olszewski², Piotr Tarkowski ${ }^{3}$, Michał Sojka ${ }^{1}$, Tomasz Jargiełło ${ }^{1}$ \\ ${ }^{1}$ Department of Interventional Radiology and Neuroradiology, Medical University of Lublin, Lublin, Poland \\ ${ }^{2}$ Department of Cardiosurgery, Medical University of Lublin, Lublin, Poland \\ ${ }^{3}$ Department of Radiology and Nuclear Medicine, Medical University of Lublin, Lublin, Poland
}

Adv Interv Cardiol 2020; 16, 4 (62): 519-520

DOI: https://doi.org/10.5114/aic.2020.101784

Transcatheter aortic valve implantation (TAVI) is a minimally invasive alternative to open-heart surgery for highrisk patients with severe symptomatic aortic stenosis. However, with reported incidence of 5-10\%, acute ischemic stroke remains a serious complication significantly increasing morbidity and mortality among patients undergoing a TAVI procedure [1]. Therefore, several cerebral embolic protection devices aiming to prevent procedural debris reaching the cerebral circulation were introduced. Nevertheless, if ischemic stroke should occur, immediate mechanical thrombectomy is reported to be the most effective therapeutic option [2].

A 77-year-old man with symptomatic high-grade aortic stenosis (EuroSCORE 4.61\%) was admitted to the Cardiosurgery Department for operative treatment. Due to a history of triple coronary artery bypass graft surgery and percutaneous coronary interventions he was qualified for a TAVI procedure. Under all sterile conditions and in local anesthesia with analgosedation both right (surgical) and left (percutaneous) femoral access was obtained and pre-implantation balloon aortic valvuloplasty performed. After valvuloplasty, the symptoms of a left-hemispheric stroke (decreased level of consciousness, aphasia, partial gaze palsy, hemianopia and hemiparesis) were observed. The patient's National Institutes of Health Stroke Scale (NIHSS) score was 12. Immediate cerebrovascular angiography was performed by an on-call neuroradiologist. It revealed occlusion of the left middle cerebral artery (MCA) at the proximal M1 segment (Figure $1 \mathrm{~B}$ ). Mechanical thrombectomy was performed with an aspiration device (Penumbra, CA, US). Complete recanalization of the vessel marked as Thrombolysis in Cerebral Infarction ( $\mathrm{TICl}) 3$ was confirmed in follow-up angiography (Figure $1 \mathrm{C}$ ). Afterwards, ACURATE neo $L$ valve (Boston Scientific, MA, US) was deployed with no complications. Control angiography and transesophageal echocardiogram (TEE) confirmed the correct position of the valve (Figure $1 \mathrm{D}$ ).

After the procedure the patient's condition improved. Control noncontrast computed tomography performed 24 hours after thrombectomy ruled out hemorrhagic transformation of infarction. After 10 days of hospitalization he was discharged with no signs of paralysis but persistent aphasia, minor facial paralysis, partial hemianopia, mild sensory loss and inattention on one side (NIHSS 8). TEE at discharge showed significant improvement: mean aortic gradient - $10 \mathrm{~mm} \mathrm{Hg}$, maximal aortic gradient $-16 \mathrm{~mm} \mathrm{Hg}$. Three months later further improvement of clinical condition was observed (modified Rankin Scale score 2, NIHSS 6 - minor facial paralysis, aphasia, partial hemianopia, mild right sided sensory loss and inattention).

Migration of procedural debris to the cerebral circulation can occur at any stage of the TAVI procedure. In the described case symptoms of acute ischemia were observed after valvuloplasty preceding valve implantation. According to Grube et al. direct TAVI technique performed without pre-dilatation may result in a lower rate of cerebrovascular events [3]. On the other hand, Van Mieghem et al. found that the number and volume of cerebral ischemic lesions on post-procedural diffusion-weighted MRI was higher among patients after valve implantation without prior balloon valvuloplasty compared to those who had pre-dilatation [4]. Valve positioning and implantation are other high-risk moments of the procedure. Regardless of the stage of the procedure at which cerebral embolization occurs, immediate diagnosis followed by mechanical thrombectomy gives a chance to rescue the 

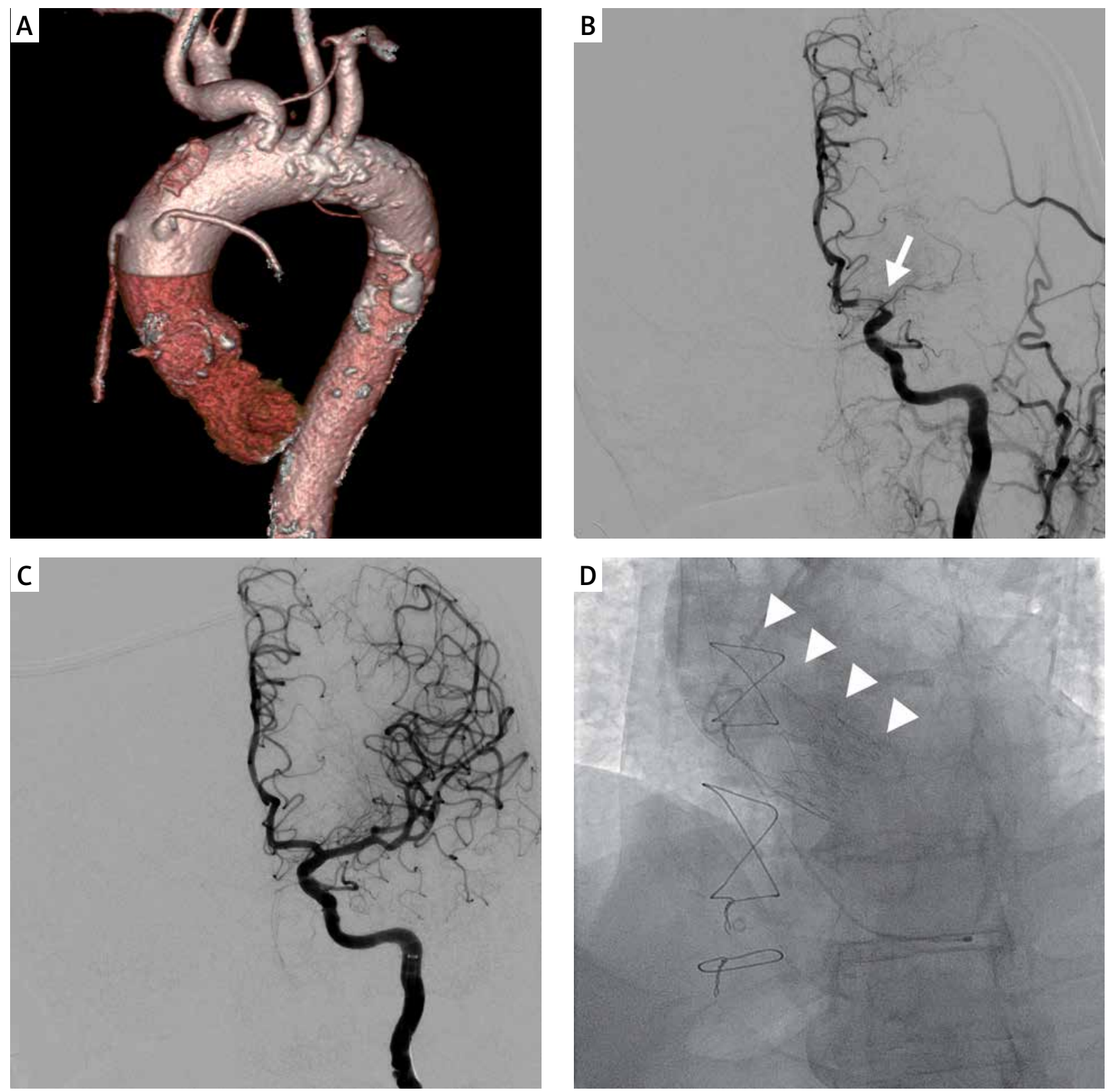

Figure 1. A - Calcifications in sinotubular junction, aortic arch and descending aorta visible on pre-procedural computed tomography. Aortic valve calcification Agatston score was 2666. B - Cerebral angiography revealing occlusion of the left MCA at the proximal M1 segment (arrow). C - Follow-up angiography after mechanical thrombectomy confirming complete recanalization of left MCA. D - Final angiography showing correct position of the ACURATE neo L valve

area of ischemic penumbra, which increases the probability of a favorable clinical outcome.

\section{Conflict of interest}

The authors declare no conflict of interest.

\section{References}

1. Seeger J, Kapadia SR, Kodali S, et al. Rate of peri-procedural stroke observed with cerebral embolic protection during transcatheter aortic valve replacement: a patient-level propensity-matched analysis. Eur Heart J 2018; 40: 1334-40.
2. Teitelbaum M, Kotronias RA, Sposato L, et al. Cerebral embolic protection in TAVI: friend or foe. Interv Cardiol 2019; 14: 225.

3. Grube E, Naber C, Abizaid A, et al. Feasibility of transcatheter aortic valve implantation without balloon pre-dilation: a pilot study. JACC Cardiovasc Interv 2011; 4: 751-7.

4. Van Mieghem NM, Schipper ME, Ladich E, et al. Histopathology of embolic debris captured during transcatheter aortic valve replacement. Circulation 2013; 127: 2194-201. 